Case Report

\title{
Transanal Total Mesorectal Excision (Ta-TME) in a Rectal Cancer Patient with a History of Abdominal Surgery: A Case Report
}

\author{
Satoshi Narihiro, Hironori Ohdaira, Hideyuki Takeuchi, Teppei Kamada, Rui Marukuchi, \\ Norihiko Suzuki, Sojun Hoshimoto, Masashi Yoshida and Yutaka Suzuki \\ Department of Surgery, International University of Health and Welfare Hospital, Nasushiobara, Japan
}

\begin{abstract}
A 65-year-old man was followed up after undergoing Hartmann's operation for the treatment of obstructive colon cancer 1 year earlier. He presented with bloody stool and underwent examination, including lower gastrointestinal endoscopy, and he was diagnosed with rectal cancer. Since he had a history of multiple abdominal surgeries, including Hartmann's operation, severe pelvic adhesions were expected. Thus, in consideration of surgical safety and curability, transanal total mesorectal excision (Ta-TME) was performed. The duration of the surgery was $3 \mathrm{~h}$, and there was minimal blood loss. Histopathological findings did not reveal remnants of cancer in the resected margin, and the patient was discharged on hospital day 7. Rectal cancer has a higher rate of local recurrence than colon cancer. To prevent local recurrence, ensuring a rectal circumferential resection margin (CRM) with TME is essential, which is, however, challenging in obese patients and in those with giant tumors, contracted pelvis, prostatic hypertrophy, etc., since these conditions complicate pelvic surgery. The same is true for patients with a history of multiple abdominal surgeries. It is expected that these problems can be resolved by Ta-TME. In the present case, Ta-TME was extremely useful in rectal cancer surgery for a patient with a history of multiple abdominal surgeries, including Hartmann's operation.
\end{abstract}

\section{Keywords}

transanal total mesorectal excision, Hartmann's operation, rectal cancer

J Anus Rectum Colon 2020; 4(1): 41-46

\section{Introduction}

Rectal cancer has a higher rate of local recurrence than colon cancer[1]. In order to prevent such local recurrence following rectal cancer surgery, ensuring a circumferential resection margin (CRM) with total mesorectal excision (TME) is important[2-5]; however, with the traditional transabdominal approach, it is difficult to ensure a CRM with TME when pelvic surgery is problematic, as in obese patients and those with giant tumors, contracted pelvis, or prostatic hypertrophy[6,7]. Transanal (Ta)-TME is considered to resolve these issues. Compared with the transab- dominal approach, Ta-TME is an easier surgical procedure in cases with anticipated difficult pelvic surgery and has been thought to be useful from the perspective of ensuring a CRM with TME[8]. A case of Ta-TME that was performed in a rectal cancer patient with a history of multiple abdominal surgeries, including Hartmann's operation, is presented.

\section{Case Report}

A 65-year-old man presented to our hospital with anemia and abdominal fullness 1 year earlier. Based on the results of lower intestinal tract endoscopy and the CT findings, he 


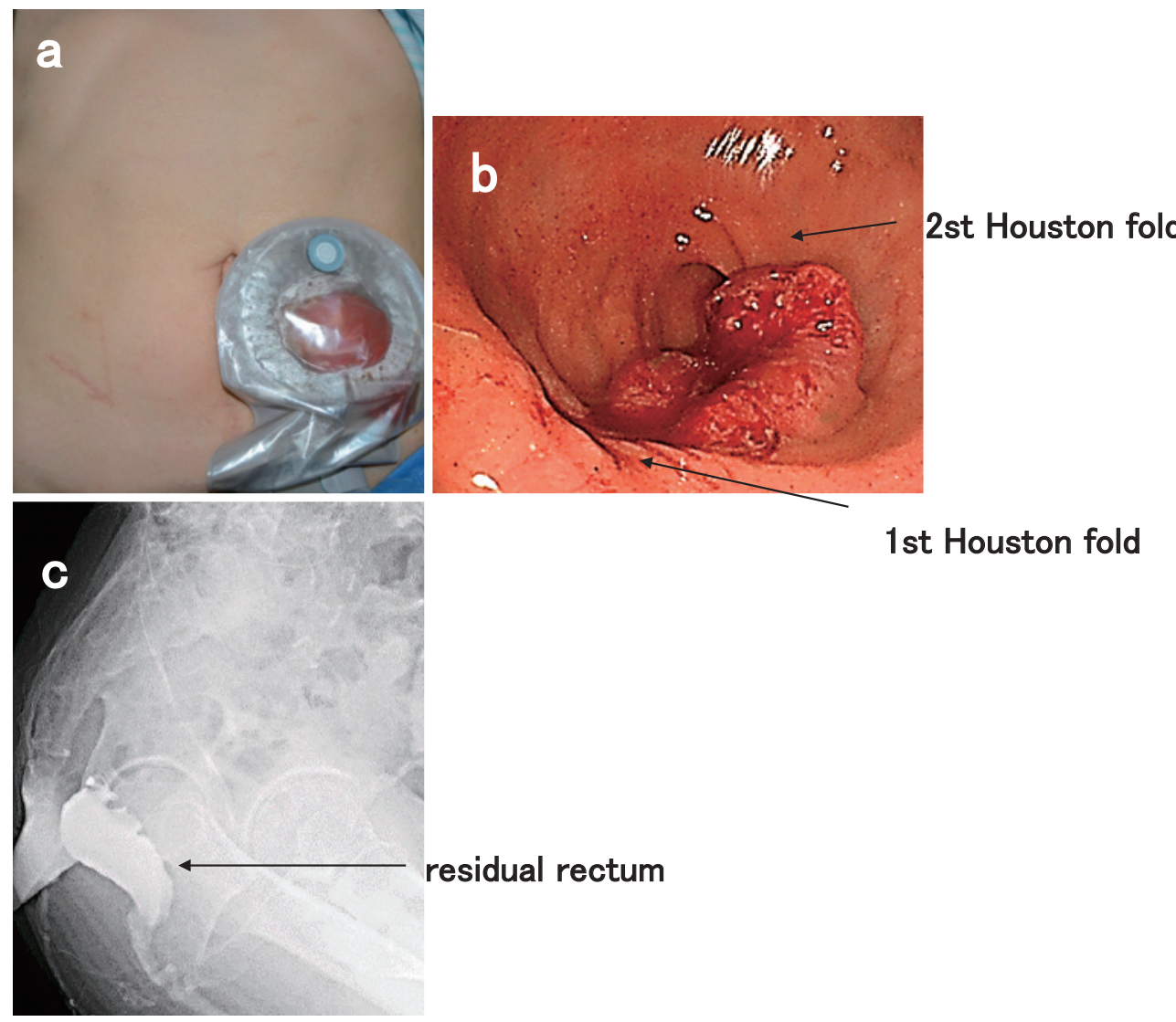

Figure 1. The tumor was present in the Rb-expressing area of the rectum, and the remaining rectum was about $6-\mathrm{cm}$ long. The patient had previously undergone multiple abdominal operations, including open Hartmann's operation for obstructive colorectal cancer, appendicectomy, and laparoscopic cholecystectomy.

was diagnosed with obstructive cancer of the sigmoid colon. An emergency operation was performed on the same day, because we could not pass the scope through the intestinal lumen, and transanal decompression was difficult. He underwent open Hartmann's operation, and was followed up since then. He also had a history of laparoscopic cholecystectomy 5 years earlier and appendectomy 10 years earlier. One year after Hartmann's operation, he underwent comprehensive examination for bloody stools originating from the remaining rectum, which led to a diagnosis of rectal cancer ( $\mathrm{Rb}$ type 2 , $20 \mathrm{~mm}$, cT2 cN0 cM0 stage I); hence, surgery was planned.

On physical examination, a single stoma at the lower left abdomen and a skin incision mark and a port wound at the lower right abdomen were observed (Figure 1a).

Lower gastrointestinal endoscopy revealed an approximately $25-\mathrm{mm}$, semicircular, type 2 lesion in the rectal $(\mathrm{Rb})$ region (Figure 1b). Abdominal computed tomography revealed no distant and lymph node metastases. Identification of the primary lesion was also difficult. Barium enema examination revealed that the length of the remaining rectum was approximately $6 \mathrm{~cm}$ (Figure 1c).

Since severe pelvic adhesions were expected, we decided to try performing surgery using the transanal approach. Intraoperatively, the remaining intestinal tract, including the previous staple line on the oral side of the tumor, was excised, and the incision line was set at $3 \mathrm{~cm}$ from the tumor on the anal side. To prevent intraoperative dissemination of the tumor cells, purse-string sutures were used at an appropriate distance from the tumor (Figure 2a), and a fullthickness incision was started from the posterior wall to expose the longitudinal muscle. Then, the longitudinal muscle was incised, and detachment was performed while verifying the presence of the levator ani muscle on the dorsal side; the hiatal ligament was confirmed and subsequently dissected (Figure 2b). Subsequently, dissection of the anterior wall was performed. In order to protect the prostate, detachment was performed at the dorsal sparse layer.

Subsequently, the rectourethralis muscle was confirmed and dissected (Figure 2c), and detachment was advanced as far cranially as possible. Finally, the walls on both sides were detached. After confirming the left and right neurovascular bundles (NVBs), the space between the fascia propria recti and NVBs was detached, and the anterior and posterior walls were connected. Due to the prior surgery, a portion of 

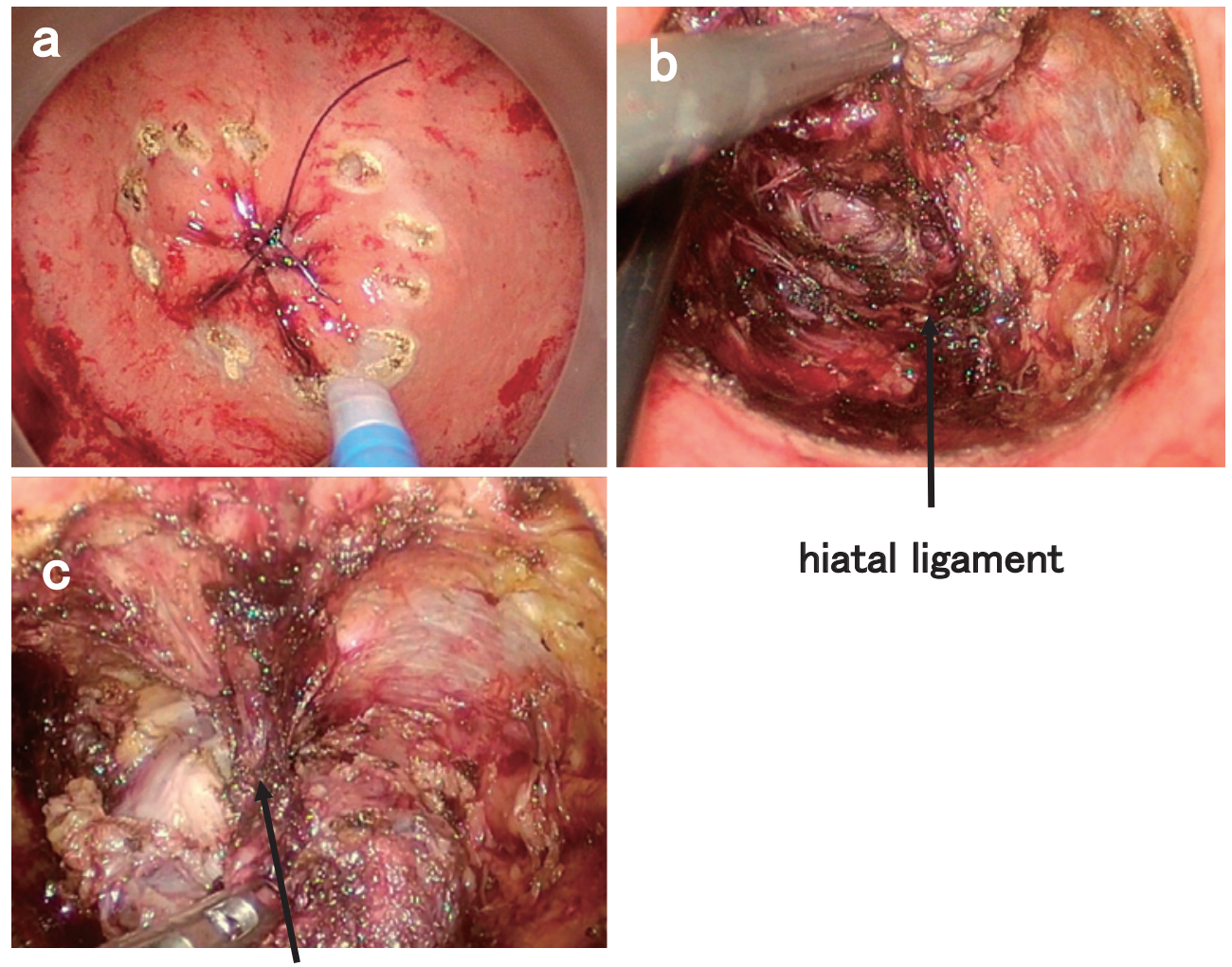

hiatal ligament

\section{rectourethral muscle}

Figure 2.

a) The intestinal tract was closed at an appropriate distance from the tumor to prevent dissemination of tumor cells.

b) The posterior wall was detached from the hiatal ligament.

c) We confirmed that the prostate and the rectourethralis muscle was cut after confirming the position of the prostate.

the detached layer of the posterior wall was unclear; however, TME could be completed up to the suture line (staple line) of the previous surgery (Figure 3). After the absence of organ injury was confirmed and hemostasis was achieved, the abdominal operation was commenced. In this step, detachment was difficult to perform due to strong adhesions of the small intestines; however, because thorough detachment was performed from the perineal side, pelvic operation could be completed in a short time. For reconstruction, although it was possible to preserve the anal sphincter, we decided not to perform anastomosis, because the intestinal tract from the sigmoid colon to the descending colon was short, and there were numerous adhesions around the stoma. After extraction of the specimen, perineal plication was performed as much as possible without anastomosis, concluding the surgery. The duration of the surgery was $3 \mathrm{~h}$, and there was minimal blood loss.

On pathological examination of the resected specimen, the tumor was observed to be Rb type $218 \times 17 \mathrm{~mm}$ pT1b pN0 pM0 pPM0 pDM0 pRM0, and the distal margin was $2 \mathrm{~cm}$ long and did not contain cancer remnants (Figure 4). The patient made satisfactory progress postoperatively and was discharged on hospital day 7.

\section{Discussion}

With the development of procedures such as anal function-preserving surgery, laparoscopic surgery, and robotic surgery, the surgical treatment of rectal cancer has changed dramatically in recent years. Rectal cancer has a higher rate of local recurrence than colon cancer. In order to prevent postoperative local recurrence, preoperative radiation therapy and lateral lymph node dissection have been proposed both in Japan and in other countries. In the surgical procedure, ensuring a CRM with TME is essential to decrease the rate of local recurrence. However, ensuring a CRM with TME performed via the traditional transabdominal approach is not technically easy and is oncologically problematic in obese patients and in those with giant tumors, contracted pelvis, or prostatic hypertrophy. Ta-TME was first reported by Sylla et al. in 2010[9], and its use has become widespread in Japan, primarily for the treatment of 


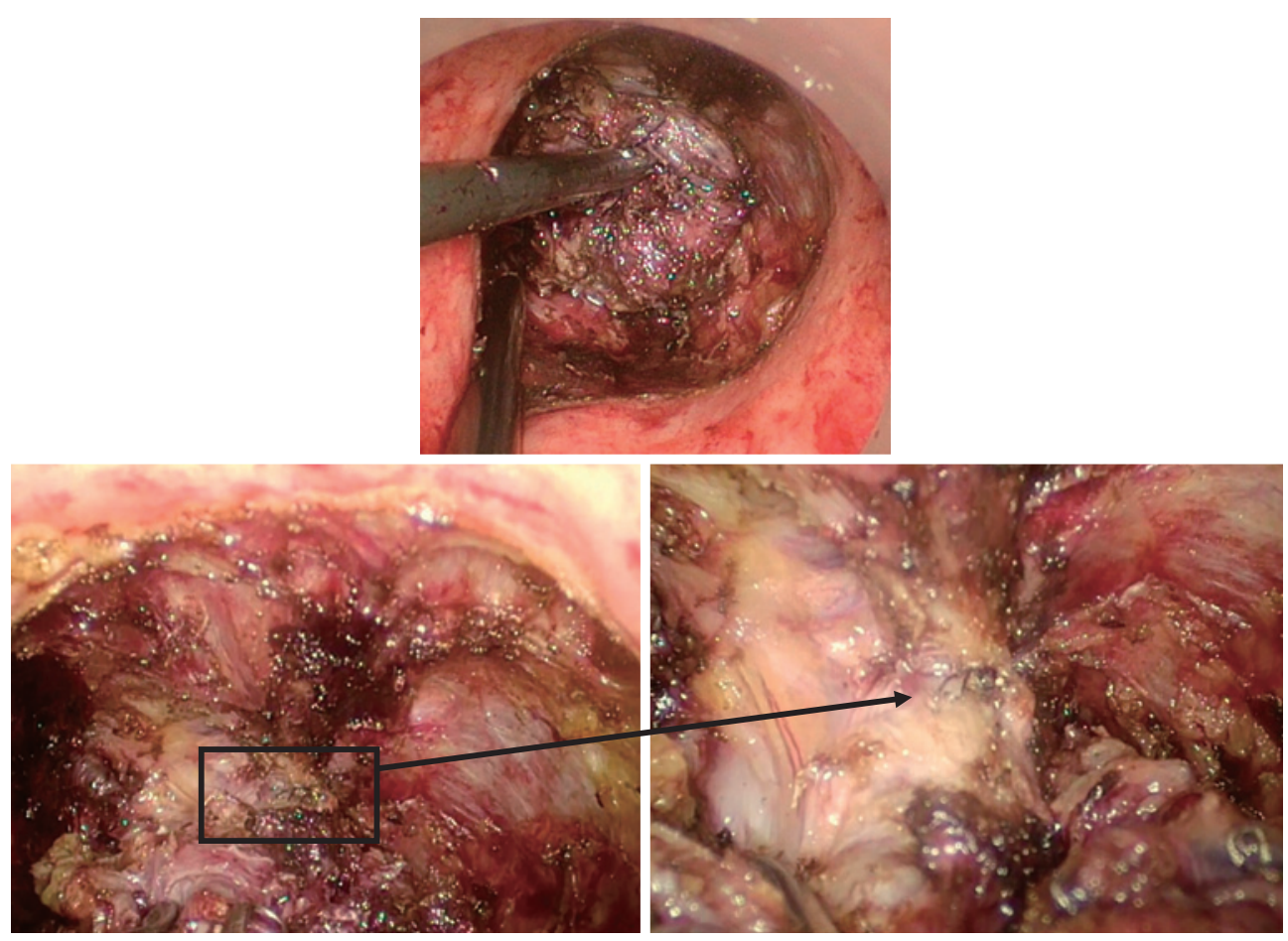

Figure 3. TME was performed all the way to the suture line (staple line) of the previous operation. The rectangle indicates the previous suture line (staple line).

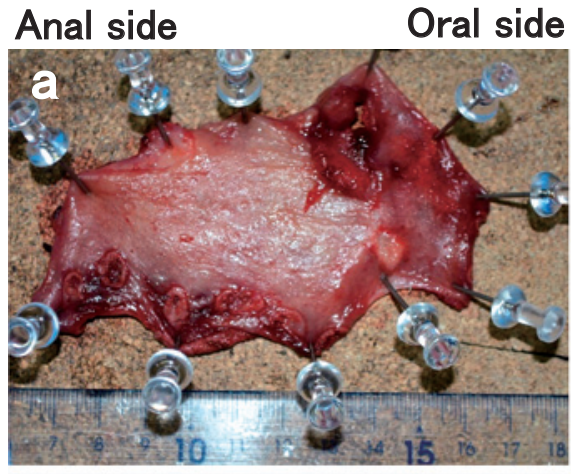

c

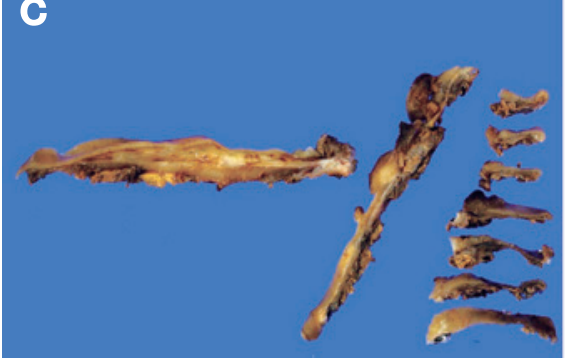

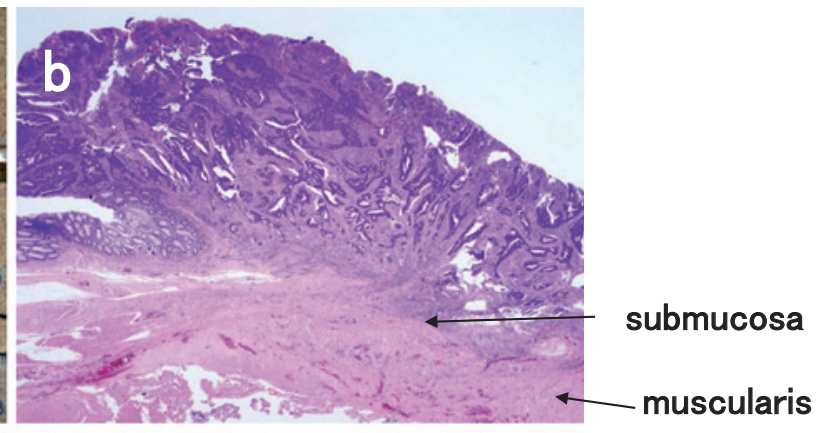

propria

Figure 4. The pathological findings were Rb type $218 \times 17 \mathrm{~mm}$ pT1 pN0 pM0. The cancer had penetrated the submucosa (hematoxylin-eosin stain, 40x). The stump was negative for cancer.

lower rectal cancer. Ta-TME is anticipated to be useful from the perspective of improving surgical maneuverability and decreasing the rate of local recurrence by ensuring a CRM with TME in patients who are likely to be difficult candi- dates for pelvic surgery for the reasons mentioned above[10-12]. Ta-TME has a short history and is currently considered to be a type of advanced surgery; thus, there are virtually no reports related to Ta-TME or case reports of the 
procedure being performed in patients with a history of multiple abdominal surgeries, as in the present case.

In Ta-TME, TME is performed under a direct and magnified transanal surgical view, and the procedure allows greater maneuverability of the device and forceps. Moreover, with a two-team approach, the surgery can be performed in a coordinated manner from both the abdominal cavity and the perineal side. Ta-TME is considered to be a useful procedure in rectal cancer patients with a history of multiple abdominal surgeries and also in those likely to have surgical difficulties using the transabdominal approach. The present case, a lower rectal cancer patient with a history of multiple abdominal surgeries, including Hartmann's operation, had risks of bleeding and organ injury during pelvic surgery due to adhesions and unclear anatomic structures around the pelvis. Poor surgical maneuverability and loss of curability were also potential problems. Another characteristic of TaTME is that it can be performed using a two-team approach, working in a coordinated manner from the abdominal cavity and the perineal side; however, in the present case, a transanal approach was adopted as much as possible, which enabled an easier abdominal operation in a shorter amount of time, consequently minimizing the duration of surgery and blood loss. In the present case, we took advantage of TaTME and decided to use the Ta-TME approach and performed a large part of the dissection transanally. The traditional transabdominal approach might have resulted in a longer duration of surgery, increased blood loss, poor visual field due to adhesion detachment, incomplete TME, and increased risk of local recurrence from an inappropriate CRM. From both surgical safety and oncological perspectives, TaTME selection as the surgical procedure of choice was appropriate in the present case. There was a possibility of anal reconstruction and anal function preservation in the present case, but we did not perform anal reconstruction considering that the surgical invasion is bigger. If patients want to preserve anal function and undergo anal reconstruction, we had to plan the reconstruction with the mobilization of splenic flexure and using transverse colon.

Since this was the first Ta-TME case performed at our hospital, we invited an instructor to provide surgical support according to the guidelines of the Ta-TME introduction curriculum[13]. The Ta-TME technique was approved by the clinical ethics committee (No. 2019-4) at our hospital in July 2019. Currently, we are planning to accumulate more such cases after obtaining the approval of our Institutional Review Board. Our surgical indication is one of the topics for future study.

The present case was a patient who had previously undergone surgery for obstructive colon cancer, which accounts for $10 \%$ of all colon cancer cases. Patients with obstructive colon cancer cannot undergo preoperative lower gastrointestinal endoscopy, and approximately $10 \%$ of them are known to present with multiple synchronous or metachronous colon cancers[14]. Recently, the use of colonic stents has become popular, making it possible to perform an anastomosis with one-stage resection after decompression. However, most surgeries are performed in cases with anastomotic difficulties, and Hartmann's operation is commonly selected for left colon to rectal cancers[15]; thus, it is highly likely that problems, as described in the present case, will occur in other cases as well.

As shown in the present case, Ta-TME is a useful method even when rectal cancer occurs immediately following Hartmann's operation. Although Ta-TME is a useful procedure in rectal cancer surgery, a more comprehensive examination of surgical techniques, anatomical understanding, etc., is necessary in the future. Numerous problems remain, including complications such as intraoperative urethral injury and postoperative anastomotic leakage. Ongoing analysis of this procedure is therefore required.

\section{Conflicts of Interest}

There are no conflicts of interest.

\section{Author Contributions}

SN made substantial contributions to study conception and design, acquisition of data, and analysis and interpretation of data.

HO was involved in drafting the manuscript and revising it critically for important intellectual content.

HT took part in the discussions about this study.

TK took part in the discussions about this study.

RM took part in the discussions about this study.

NS took part in the discussions about this study.

SH took part in the discussions about this study.

MY took part in the discussions about this study.

YS gave the final approval for the version to be published.

All the authors read and approved the final manuscript.

\section{Consent}

We obtained the patient's consent for publication of this report.

\section{References}

1. Heald RJ, Husband EM, Ryall RD. The mesorectum in rectal cancer surgery-the clue to pelvic recurrence? Br J Surg. 1982 Oct;69 (10):613-6.

2. Heald RJ, Ryall RD. Recurrence and survival after total mesorectal excision for rectal cancer. Lancet. 1986 Jun;1(8496):1479-82.

3. Park JS, Huh JW, Park YA, et al. A circumferential resection margin of $1 \mathrm{~mm}$ is a negative prognostic factor in rectal cancer patients with and without neoadjuvant chemoradiotherapy. Dis Colon Rectum. 2014;57:933-40.

4. Nagtegaal ID, Marijnen CA, Kranenbarg EK, et al. Circumferential margin involvement is still an important predictor of local re- 
currence in rectal carcinoma: not one millimeter but two millimeters is the limit. Am J Surg Pathol. 2002;26:350-7.

5. Hwang MR, Park JW, Park S, et al. Prognostic impact of circumferential resection margin in rectal cancer treated with preoperative chemoradiotherapy. Ann Surg Oncol. 2014;21:1345-51.

6. Hamabe A, Okita K, Nishidate T, et al. TaTME for rectal cancer in obesity patients. Surgery. 2019; 81: 250-58.

7. Hasegawa H, Tsukada Y, Sasaki T, et al. Laparoscopic ISR combined with taTME. Operation. 2018;72:1849-60.

8. Hasegawa H, Okabayashi K, Tsuruta M, et al. Evolution of surgery for rectal cancer: Transanal total mesorectal excision $\sim$ new standard or fad. J Anus, Rectum Colon. 2018;2(4):115-21.

9. Sylla P, Rattner DW, Delgado S, et al. NOTES transanal rectal cancer resection using transanal endoscopic microsurgery and laparoscopic assistance. Surg Endosc. 2010;24(5):1205-10.

10. Bonjer HJ, Deijen CL, Abis GA, et al. COLOR II study group: A randomized trial of laparoscopic versus open surgery for rectal cancer. N Engl J Med. 2015;372(14):1324-32.

11. Jeong SY, Park JW, Nam BH, et al. Open versus laparoscopic surgery for mid-rectal or low-rectal cancer after neoadjuvant chemoradiotherapy (COLEAN trial): survival outcomes of an open-label, non-inferiority, randomised controlled trial. Lancet Oncol. 2014;15
(7):767-74.

12. Stevenson AR, Solomon MJ, Lumley JW, et al. Effect of laparoscopic-assisted resection vs open resection on pathological outcomes in rectal cancer: The AlaCaRT randomized clinical trial. JAMA. 2015;314(13):1356-63.

13. Francis N, Penna M, Mackenzie H, et al. Consensus on structured training curriculum for transanal total mesorectal excision (TaTME). Surg Endscop. 2017;31(7):2711-9.

14. Kashimura S, Yoshino Y, Sanpei M, et al. Outcomes of bridge to surgery for obstructive colorectal cancer after metallic stent placement in our hospital (in Japanese). Jpn J Cancer Chemother. 2016; 43(12):1641-3.

15. Rex DK, Kahi CJ, Levin B, et al. American Cancer Society; US Multi-Society Task Force on Colorectal Cancer: Guidelines for colonoscopy surveillance after cancer resection: a consensus update by the American Cancer Society and the US Multi-Society Task Force on Colorectal Cancer. Gastroenterology. 2006;130(6): 1865-71.

Journal of the Anus, Rectum and Colon is an Open Access journal distributed under the Creative Commons Attribution-NonCommercial-NoDerivatives 4.0 International License. To view the details of this license, please visit (https://creativ ecommons.org/licenses/by-nc-nd/4.0/). 